\title{
Location-Routing Problem under Emergency with Simultaneous Relief Delivery and Victim Evacuation
}

\author{
Ruoqi Du and Hong Zhou* \\ School of Economics and Management, Beihang University, Beijing 100191, China \\ ${ }^{*}$ Corresponding author
}

\begin{abstract}
The location-routing problem under emergency with simultaneous relief materials delivery and victim evacuation is studied and modeled. The number of victims is assumed ambiguous, risk preference of the decision maker is considered, and mixed transportation of relief and victims is allowed. A chance-constrained programming model is proposed based on the fuzzy credibility theory, and a genetic algorithm is designed to solve the problem.
\end{abstract}

Keywords-location-routing problem; simultaneous relief delivery and victims evacuation; fuzzy credibility; genetic algorithm

\section{INTRODUCTION}

It is an important issue in both academic research and practical management to find effective ways to reduce the consequence of the disaster, such as the earthquake. Usually after the strike of an earthquake, efforts are mainly focused on rescuing the injured people and dispatching reliefs to the affected areas from the Emergency Logistics Centers (ELCs). Hence how to determine the proper location for an ELC and how to plan the routes for the vehicles of relief delivery become essential and urgent. The facility location problem (FLP) and vehicle routing problem (VRP) have been studied for many years. Since the number and locations of ELCs directly influence the vehicle routing while the efficient vehicle routing strategies also have some requirements on the location of facilities, it will take advantages sometimes to consider FLP and VRP jointly. Thus the location routing problem (LRP) has aroused more and more attentions recently [1]. To increase the efficiency of transportation, simultaneous pickup and delivery (SPD) are usually encouraged, and the relative problems have received great concerns, see Wang et al [2].

The severity of disaster effects is usually unknown in the initial period, hence decisions have to be made under uncertainty. Fuzzy set theory has been widely adopted to process the uncertain information in logistics management, such as the uncertain demand in VRP [3], and the chanceconstrained programming model has been frequently employed (see [4][5]). Previously, fuzzy possibility was often adopted to deal with fuzzy demand, but it has no self-dual property. Liu introduced the concept of fuzzy credibility in order to overcome this shortage [6], and was adopted in this paper.

The rest of the paper is organized as follows. The model for location-routing problem with simultaneous pickup and delivery (LRPSPD) under uncertainty was formulated in Section 2, which considered the delivery of emergency supplies and evacuation of victims simultaneously. In Section 3, a genetic algorithm was designed. Then numerical experiments were conducted and the results were analyzed in Section 4. Finally in Section 5, some conclusions were summarized.

\section{FUZZY LRPSPD MODEL}

\section{A. Problem Description}

First, the problem considered in this paper has the following features. There are some warehouses separately located and can be used as Emergency Logistics Centers (ELCs) in case of occurring of an emergent event. And some shelters or hospitals are also distributed in this area and available for receiving victims. When an earthquake occurs, some areas will be affected and need emergent rescue, known as demand nodes.

Decision makers have to decide which warehouses will be selected as ELCs and how to distribute the reliefs to the affected areas based on the priority in severity, required due time, decision makers' preferences, etc.. Besides, victims, especially the injured people, in the affected areas have to be transferred to proper shelters or hospitals as well. We assume that the victims can be picked up by the same vehicle which delivers reliefs. The number of victims is represented with a triangle fuzzy number $(\alpha, \beta, \gamma)$. The goal is to determine the quantity and locations of ELCs, and to find the best routes for delivering reliefs to the affected areas meanwhile transferring the victims to hospitals, in minimizing the total cost.

\section{B. Mathematical Model}

Some assumptions are made as following: (1) each demand node is visited exactly once by a vehicle, and the service time is ignored; (2) the victims can be carried on the same vehicle together with reliefs; (3) a vehicle will start from an ELC and stop at a hospital without going back to the ELC after visiting the final demand node on the route; (4) it is allowable that more than one vehicle start from an ELC; (5) the total load of a vehicle (including reliefs and victims) cannot exceed its capacity; (6) no restriction on the capacity of hospital.

Notations are defined as follows.

1) Sets:

$\boldsymbol{P} \quad$ Set of all potential ELCs $\quad \boldsymbol{P}=\{p \mid p=1,2, \ldots, P\}$

A Set of demand nodes $\quad \boldsymbol{A}=\{i \mid i=1,2, \ldots, N\}$

$\boldsymbol{H}$ Set of hospitals $\quad \boldsymbol{H}=\{h \mid h=1,2, \ldots, H\}$ 
$\begin{array}{cl}\boldsymbol{K} & \text { Set of vehicles } \quad \boldsymbol{K}=\{k \mid k=1,2, \ldots, K\} \\ 2) \quad & \text { Parameters: } \\ f_{p} & \text { Setup cost for employing the } p \text { th warehouse as an } \\ \text { ELC } & \\ S_{p} & \text { Supply of the } p \text { th warehouse as an ELC } \\ C V_{k} & \text { Capacity of vehicle } k \\ c_{k} & \text { Unit transportation cost (per kilometer) of vehicle } k \\ w_{k} & \text { Fixed cost for vehicle } k \text { if employed } \\ d_{i j} & \text { Distance between node } i \text { and } j \\ W & \text { Capacity occupation by a victim } \\ t_{i j} & \text { Travel time from node } i \text { to } j \\ T_{j} & \text { Arrival time of a vehicle at node } j, \text { where } T_{j}=0 \forall j \in \boldsymbol{P} \\ T_{\max } & \text { The allowable latest arrival time for each demand }\end{array}$ node

$D_{j} \quad$ Demand for relief materials at node $j, j \in \boldsymbol{A}$

$\tilde{b}_{j}$ Quantity of victims at node $j$ estimated in fuzzy number $\tilde{b}_{j}=\left(\alpha_{j}, \beta_{j}, \gamma_{j}\right)$ $j \in \boldsymbol{A}$

$\tilde{U}_{k j}$ Remaining capacity of vehicle $k$ after visiting node $j$,

$D P I$ Decision maker's preference index, $D P I \in[0,1]$

$M \quad$ A big enough positive number

3) Decision variables

$y_{p} \quad 1$ if warehouse $p$ is selected as ELC; 0 otherwise

$x_{i j k} \quad 1$ if vehicle $k$ travels from node $i$ to $j$; 0 otherwise

$z_{p k} \quad 1$ if vehicle $k$ is assigned to ELC $p ; 0$ otherwise

The problem can be formulated with the following mathematical model:

$$
\text { Min Total Cost }=\sum_{p \in \boldsymbol{P}} f_{p} y_{p}+\sum_{i \in \boldsymbol{P} \cup \boldsymbol{A}} \sum_{j \in \boldsymbol{H} \cup \boldsymbol{A}} \sum_{k \in \boldsymbol{K}} c_{k} d_{i j} x_{i j k}+\sum_{p \in \boldsymbol{P}} \sum_{k \in \boldsymbol{K}} w_{k} z_{p k}
$$

Subject to

$$
\begin{gathered}
\sum_{j \in \boldsymbol{A}} \sum_{k \in \boldsymbol{K}} x_{p j k} \leq y_{p} \times K \quad \forall p \in \boldsymbol{P} \\
\sum_{k \in \boldsymbol{K}} z_{p k} \leq y_{p} \times K \quad \forall p \in \boldsymbol{P} \\
\sum_{j \in \boldsymbol{A}} x_{p j k}=z_{p k} \quad \forall p \in \boldsymbol{P}, \forall k \in \boldsymbol{K}
\end{gathered}
$$

$$
\begin{gathered}
\sum_{j \in \boldsymbol{P} \cup \boldsymbol{A}} x_{j i k}-\sum_{j \in \boldsymbol{P} \cup \boldsymbol{A}} x_{i j k}=0 \quad \forall i \in \boldsymbol{A}, \forall k \in \boldsymbol{K} \\
\sum_{j \in \boldsymbol{P} \cup \boldsymbol{A}} \sum_{k \in \boldsymbol{K}} x_{h j k}=0 \quad \forall h \in \boldsymbol{H} \\
\sum_{j \in \boldsymbol{P} \cup \boldsymbol{A}} \sum_{k \in \boldsymbol{K}} x_{i j k}=1 \quad \forall j \in \boldsymbol{A} \\
\sum_{i \in \boldsymbol{S}} \sum_{j \in S} x_{i j k} \leq|\boldsymbol{S}|-1 \quad \forall \boldsymbol{S} \subseteq \boldsymbol{A}, \forall k \in \boldsymbol{K} \\
T_{i}+t_{i j}-T_{j} \leq\left(1-\sum_{k \in \boldsymbol{K}} x_{i j k}\right) M \quad \forall i \in \boldsymbol{P} \cup \boldsymbol{A}, \forall j \in \boldsymbol{H} \cup \boldsymbol{A}
\end{gathered}
$$

$$
\sum_{k \in \mathbf{K}} z_{p k}\left(\sum_{i \in \boldsymbol{P} \cup \boldsymbol{A}} \sum_{j \in \boldsymbol{A}} D_{j} x_{i j k}\right) \leq S_{p} y_{p} \forall p \in \boldsymbol{P}
$$

$$
\tilde{U}_{k j} \geq \tilde{U}_{k i}+D_{j}-\tilde{b}_{j} \times W-\left(1-x_{i j k}\right) M \forall i \in \boldsymbol{P} \cup \boldsymbol{A}, \forall j \in \mathbf{A}, \forall k \in \boldsymbol{K}
$$

$$
\operatorname{Cr}\left\{\tilde{U}_{k j} \geq 0\right\} \geq D P I \quad \forall j \in \boldsymbol{A}
$$

$$
x_{i j k} \in\{0,1\} \quad \forall i \in \boldsymbol{P} \cup \boldsymbol{A}, \forall j \in \boldsymbol{H} \cup \boldsymbol{A}, \forall k \in \boldsymbol{K}
$$

$$
z_{p k} \in\{0,1\} \forall p \in \boldsymbol{P}, \forall k \in \boldsymbol{K}
$$

$$
y_{p} \in\{0,1\} \forall p \in \boldsymbol{P}
$$

In above model, the objective to be minimized includes the setup cost for ELCs, transportation cost, as well as the fixed cost for vehicles. Constraint (2) and (3) indicate that vehicles can be assigned to warehouse $p$ only when it is selected as an ELC, while constraint (4) states that a vehicle can leave ELC $p$ only when assigned to it. Constraint (5) is the flow conservation equation. Constraint (6) specifies that vehicles will finally stop at hospitals. Constraint (7) restricts that each demand node is visited once and only once. Constraint (8) is to 
eliminate subtours from the solution. Constraint (9) represents the visiting sequence of the nodes in terms of arrival times. Constraint (10) guarantees that the arrival time at each demand node cannot be later than the specified due time. Constraint (11) and (12) are common capacity restrictions, while constraint (13) explains that the service capability of picking up victims depends on its remaining capacity along with the tour. Considering that the estimated quantity of victims is a fuzzy number, the remaining capacity of a vehicle on its tour is obviously a fuzzy number as well. The initial value for $\tilde{U}_{k j}(j \in \boldsymbol{P})$ is a non-fuzzy number which is calculated by $\tilde{U}_{k p}=C V_{k}-\sum_{i \in \mathbf{P} \cup \boldsymbol{A}} \sum_{j \in A} x_{i j k} D_{j}$. Constraint (14) reflects the risk preference of the decision maker in terms of fuzzy credibility.

Now we introduce the calculation for fuzzy credibility in constraint (14). Suppose that the route for vehicle $k$ is $\left(p, j_{1}\right.$, $\left.j_{2} \ldots, j_{k}, h\right)$, where $p$ is the ELC as its start, $h$ is the hospital at which it stops, and $j_{1}, \ldots, j_{k}$ are demand nodes it serves. The remaining capacity of vehicle $k$ after visiting $j_{i}$ can be calculated as $\tilde{U}_{k j_{i}}=\tilde{U}_{k p}+\sum_{j=j_{1}}^{j_{i}}\left(D_{j}-\tilde{b}_{j} \times W\right)=\left(\varphi_{j_{i}}, \omega_{j_{i}}, \tau_{j_{i}}\right) \quad$ where $\varphi_{j_{i}}=\tilde{U}_{k p}+\sum_{j=j_{1}}^{j_{i}}\left(D_{j}-\gamma_{j} \times W\right) \quad, \quad \omega_{j_{i}}=\tilde{U}_{k p}+\sum_{j=j_{1}}^{j_{i}}\left(D_{j}-\beta_{j} \times W\right) \quad$ and $\tau_{j_{i}}=\tilde{U}_{h p}+\sum_{j=j_{1}}^{j_{i}}\left(D_{j}-\alpha_{j} \times W\right)$.

Based on the theory about fuzzy credibility [9], the following expression of $\operatorname{Cr}\left\{\tilde{U}_{k j_{i}} \geq 0\right\}$ can be derived

$$
\operatorname{Cr}\left\{\tilde{U}_{k j_{i}} \geq 0\right\}=\left\{\begin{array}{cc}
1 & \text { if } \varphi_{j_{i}} \geq 0 \\
\frac{\tilde{U}_{k p}+\sum_{j=j_{1}}^{j_{i}}\left(D_{j}-2 \beta_{j} \times W+\gamma_{j} \times W\right)}{2 \sum_{j=j_{i}}^{j_{i}}\left(\gamma_{j} \times W-\beta_{j} \times W\right)} & \text { if } \varphi_{j_{i}} \leq 0 \leq \omega_{j_{i}} \\
\frac{\tilde{U}_{k p}+\sum_{j=j_{i}}^{j_{i}}\left(D_{j}-\alpha_{j} \times W\right)}{2 \sum_{j=j_{1}}^{j_{i}}\left(\beta_{j} \times W-\alpha_{j} \times W\right)} & \text { if } \omega_{j_{i}} \leq 0 \leq \tau_{j_{i}} \\
0 & \text { if } \tau_{j_{i}} \leq 0
\end{array}\right.
$$

$\mathrm{Cr}$ is a measure taking value between 0 and 1 , which reflects the possibility to complete the mission. When $C r=0$, in this model, the vehicle is regarded as unable to serve the next demand node, while $C r=1$ means that it is undoubtedly capable to serve the demand node. The concept of decision maker's preference index (DPI) [7] is also adopted in the paper, which expresses the decision maker's preference towards risk. If the decision maker tends toward risk seeking, he will choose a lower DPI value; while he will choose a larger DPI value if he is inclined to be conservative.

\section{GENETIC ALGORITHM}

A kind of genetic algorithm is proposed to solve the problem in this section. The main features are designed as follows.

\section{A. Encoding}

The chromosome is composed of four segments of integers. The first segment includes $N$ (the number of demand nodes) genes of integers between 1 and $K$ (the number of vehicles), indicating the index of the vehicles assigned to demand nodes. The second segment is composed of $K$ bits of integers between 1 and $P$ (the number of ELCs), with the $i$ th gene representing the index of ELC where the $i$ th vehicle departs. The third segment, consisting of $N$ genes, provides the routes of the vehicles which appear in the first segment, and the last segment of $K$ numbers tells which hospital a vehicle will finally go to. The example in Figure 1 illustrates the encoding process.

Suppose there are 10 demand nodes, 5 vehicles, 3 ELCs, and 2 hospitals. It can be found from the first segment that number 5 appears at the $1^{\text {st }}, 3^{\text {rd }}$, and $9^{\text {th }}$ position, which implies that the demand nodes at the $1^{\text {st }}, 3^{\text {rd }}$, and $9^{\text {th }}$ position of the third segment are assigned to the $5^{\text {th }}$ vehicle, i.e. demand node 9,4 , and 6 will be served by vehicle 5 in this sequence. The value of the $5^{\text {th }}$ gene on the second segment tells us that vehicle 5 will start from ELC 2. The $5^{\text {th }}$ bit of the last segment is 1, referring to that vehicle 5 will stop at hospital 1 at last. The number 1 does not appear in the first segment, implying that vehicle 1 is not assigned to any task; so it will not stop at any hospital ( 0 is assigned to the $1^{\text {st }}$ position of the last segment).

$$
\begin{array}{|l|l|l|l|}
\hline 5-4-5-4-3-3-3-3-5-2 & 1-1-2-3-2 & 9-3-4-7-10-8-2-5-6-1 & 0-1-2-1-1 \\
\hline
\end{array}
$$

\section{FIGURE I. AN ENCODED CHROMOSOME}

\section{B. Selection, Crossover and Mutation}

Roulette wheel scheme is adopted for selection, and the probability of the chromosome being selected is proportional to its fitness value, i.e. the reciprocal of the objective value. To improve the efficiency of the algorithm, different crossover and mutation schemes are designed according to the nature of different segment. Traditional two-point crossover and singlepoint mutation are employed for segment 1 and 2. To keep the feasibility of the solution, an order-based crossover and the reversal mutation are adopted for segment 3 . The simple singlepoint crossover and mutation are used for segment 4.

\section{Initialization and Termination}

The initial population is randomly generated with all segments from their allowable ranges. The maximal evolutionary generations $U$ is set as the ending condition.

\section{NUMERCIAL EXPERIMENT}

The locations of 4 candidate ELCs, 25 demand nodes, and 2 hospitals are randomly generated in the area of square $100 \times 100$. The details can be found in Table 1 and Table 2. To simplify the experiment, we assume that there are 7 homogeneous 
vehicles, with velocity $v_{k}=60$, capacity $C V_{k}=24$, fixed cost $w_{k}=300$, unit transportation cost $c_{k}=2$. The volume of a victim and unit commodity is 0.9 and 0.05 , respectively. The allowable latest arrival time is 240 . The parameters for GA are set as follows: population size $P o p=300$, crossover rate $P_{c}=$ 0.8 , mutation rate $P_{m}=0.3$, maximum generations $U=500$.
The computational results are listed in Table 3 , by changing DPI from 0.5 to 1 . It is easy to find that the total cost significantly increases with the increase of DPI. When DPI takes the value of 0.5 or 0.7 , the number of vehicles is 6 ; while it increases to 7 when DPI becomes to 0.9 or 1 .

TABLE I. INFORMATION ABOUT AFFECTED AREAS (DEMAND NODES)

\begin{tabular}{|c|c|c|c|c|c|c|c|c|c|c|c|}
\hline Node & Location & Demand & Victims & Node & Location & Demand & Victims & Node & Location & Demand & Victims \\
\hline 1 & $(20,85)$ & 120 & $(1,4,7)$ & 10 & $(18,80)$ & 100 & $(4,6,8)$ & 19 & $(4,18)$ & 100 & $(5,6,7)$ \\
\hline 2 & $(5,45)$ & 100 & $(3,5,7)$ & 11 & $(25,30)$ & 86 & $(3,5,7)$ & 20 & $(26,35)$ & 90 & $(2,4,6)$ \\
\hline 3 & $(42,15)$ & 84 & $(4,5,6)$ & 12 & $(15,10)$ & 143 & $(6,7,8)$ & 21 & $(80,90)$ & 100 & $(2,4,6)$ \\
\hline 4 & $(38,5)$ & 165 & $(4,6,8)$ & 13 & $(45,65)$ & 78 & $(4,5,6)$ & 22 & $(72,43)$ & 113 & $(3,4,5)$ \\
\hline 6 & $(85,25)$ & 82 & $(4,6,8)$ & 15 & $(31,52)$ & 90 & $(4,6,8)$ & 24 & $(93,20)$ & 70 & $(1,3,5)$ \\
\hline 7 & $(62,80)$ & 60 & $(2,3,4)$ & 16 & $(2,60)$ & 52 & $(2,4,6)$ & 25 & $(90,90)$ & 130 & $(3,5,7)$ \\
\hline 8 & $(58,75)$ & 150 & $(8,9,10)$ & 17 & $(5,5)$ & 117 & $(3,5,7)$ & & & & \\
\hline 9 & $(50,50)$ & 182 & $(2,5,8)$ & 18 & $(57,29)$ & 138 & $(5,6,7)$ & & & & \\
\hline
\end{tabular}

TABLE II. INFORMATION ABOUT POTENTIAL ELCS AND HOSPITALS

\begin{tabular}{|c|c|c|c|c|c|c|}
\hline \multirow{2}{*}{} & \multicolumn{4}{|c|}{ ELC } & \multicolumn{2}{c|}{ Hospital } \\
\cline { 2 - 7 } & 1 & 2 & 3 & 4 & 1 & 2 \\
\hline $\begin{array}{c}\text { Locati } \\
\text { on }\end{array}$ & $(40,5)$ & $(90,70)$ & $(50,30)$ & $(20,50)$ & $(62,13)$ & $(12,60)$ \\
\hline $\begin{array}{c}\text { Setup } \\
\text { cost }\end{array}$ & 2000 & 2500 & 2500 & 3000 & & \\
\hline $\begin{array}{c}\text { Capaci } \\
\text { ty }\end{array}$ & 1500 & 1700 & 2000 & 2000 & & \\
\hline
\end{tabular}

TABLE III. COMPUTATIONAL RESULTS

\begin{tabular}{|c|c|c|c|c|}
\hline DPI & ELC & $\mathbf{N v}$ & Cost & Routes \\
\hline 0.5 & 1,2 & 6 & 7645.2 & $\begin{array}{ll}1-3-12-17-19-2 & 2-21-8-1-10-2 \\
2-5-24-23-6-22-1 & 1-11-20-18-14-1 \\
2-25-7-13-16-2-2 & 1-4-9-15-2\end{array}$ \\
\hline 0.7 & 1,2 & 6 & 7692.4 & $\begin{array}{ll}1-12-17-19-2-2 & 2-7-18-1-10-16-2 \\
2-25-21-8-13-2 & 2-22-5-6-24-23-1 \\
1-3-4-11-20-2 & 1-15-9-14-1\end{array}$ \\
\hline 0.9 & 1,2 & 7 & 8043.2 & 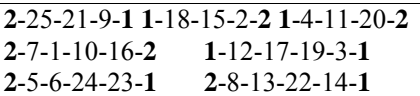 \\
\hline 1 & 1,3 & 7 & 8192.0 & $\begin{array}{lll}1-4-12-11-2 & 3-8-1-10-2 & 3-15-2-16-2 \\
3-14-5-24-23-1 & 3-22-21-25-6-1 \\
3-18-9-7-13-2 & \mathbf{1}-3-20-17-19-2\end{array}$ \\
\hline
\end{tabular}

Nv. Number of operating vehicles

The smaller DPI means that the decision maker prefers to reduce total cost and trying to make full use of vehicles by taking the risk of insufficient transportation capability, so the number of vehicles and the value of objective function are smaller but the possibility of failing to convey all the victims becomes bigger. In contrast, larger DPIs are characterized by lower utilization of vehicle capacity but higher service quality. Decision makers can make the tradeoff between risk and cost according to their preferences.

\section{CONCLUSIONS}

A LRPSPD model was developed in this paper with simultaneous relief delivery and victim evacuation. Considering the nature of information ambiguity at the early stage of the earthquake, fuzzy demand of victims was introduced and the chance-constrained method based on fuzzy credibility theory was adopted. In addition, decision maker's preference to the risk is also an important concern in our model. A genetic algorithm was designed for solving, and the results from a numerical example validated the effectiveness of the proposed model.

\section{ACKNOWLEDGMENT}

This work is supported by the Natural Science Foundation of China under Grant Number 71471007.

\section{REFERENCES}

[1] W. Yi and L. Özdamar, "A dynamic logistics coordination model for evacuation and support in disaster response activities," Eur. J. Oper. Res., vol.179, pp.1173-1193, June 2007.

[2] Y. Wang, D. C. Xu and L. J. Long, "Research of location-routing problem in emergency logistic system for post-earthquake transitional stage," J. Comput. Appl., vol.35, pp. 243-246, 2015.

[3] G. Pavkovic, "The fuzzy set theory approach to the vehicle routing problem when demand at nodes is uncertain," Fuzzy Set. \& Syst., vol. 82, pp.307-317, 1996.

[4] Z. J. Ma and Y. Dai. and S. L. Li, "Fuzzy mutli-objecitve open location-routing problem with deadlines in post-earthquake relief deliveries,” J. Syst. Manag, vol.23, pp.658-667, 2014.

[5] C. S. Liu, Y. Peng and G. Kou, "Research on fuzzy location-routing problem in post-earthquake delivery of relief materials," Chin. J. Manag. Sci., vol.24, pp:111-118, 2016.

[6] B. Liu, Theory and practice of uncertain programming, 2nd ed., Berlin: Springer-Verlag, 2009.

[7] Y. Shi, T. Boudouh and O. Grunder, "A hybrid genetic algorithm for a home health care routing problem with time window and fuzzy demand,” Expert. Syst. Appl., vol.72, pp.160-176, 2017. 